\title{
Accelerograms for building design on hard soil in Mexico City
}

\author{
M. A. Jaimes Téllez, E. Reinoso Angulo \& M. Ordaz Schroeder \\ Engineering Institute, UNAM, Mexico
}

\begin{abstract}
A method of disaggregation probabilistic seismic hazard assessment is applied to determine the most probable magnitude, $M$, and distance, $R$, of certain seismic events that can generate a fixed seismic intensity (spectral ordinate) associated with an exceedance rate for hard soil in Mexico City. The main seismic sources which affect the firm land structures are determined. In addition, the following criteria are used: (i) establishment of structural periods when the spectral ordinates should be used so that the response of the expected motion does not exceed the uniform hazard spectra and (ii) determination of the minimum number of design strong ground motions that must be considered to include spectral ordinates of uniform hazard spectra.
\end{abstract}

Keywords: subduction, intermediate depth, Mexico City, simulation, strong ground motion, design accelerograms.

\section{Introduction}

In some cases, especially when it is necessary to evaluate the response of important structures in the inelastic range, it is not sufficient to define the seismic motions by means of a design spectrum. This considers forces related to parameters of maximum response such as the spectral acceleration that does not always have a direct correlation with the loss and damage, besides not including the accumulated damage or degradation produced by the hysteretic behavior due to severe seismic events during the useful life of the structure. That is why accelerograms are used in the design of important structures, for example, to evaluate complex structures such as dams, bridges, tall buildings with irregular plant or elevation; structures where higher modes of vibration could be important or structures designed to have high demands of ductility. These accelerograms must reproduce as well as possible 
the motion expected at the site. This motion should be expressed in terms of the expected values of the magnitude, $M$, distance, $R$, the fault mechanism and the characteristics of the soil. Unfortunately, the criteria implemented by current regulations, do not contain sufficient information for engineers so they cannot use practical methods to generate design accelerograms or they use what event is available and very unlikely useful for the needed site conditions. The actual regulations and guides are vague just mentioning that accelerograms must be compatible with the design spectra and appropriate to the situation.

There are different ways to obtain the possible time histories for hard soil in Mexico City that take into account some characteristics of the ground. Here are the most common ones: (1) The first and most obvious is the use of real records available in worldwide databases [1] with similar characteristics; this means that the seismic event should have approximately the same magnitude and distance. (2) The second way is to scale real accelerograms from the site to a required level of intensity; however, these accelerograms may present the unsuitability arising from the scaling only considering the amplitude since it is very difficult to take into account the magnitude and distance with the respective change in the frequency content and duration of motion. (3) The third way is to use simulated earthquake motions compatible with a prescribed response or design spectra. In this method, it is necessary to generate a seismic motion compatible with the spectrum. However, it is known that these time histories do not have the appearance of real earthquake motion, and when the structure is subjected to them, the demands on displacements and input energy are overestimated [2]. (4) The fourth way is to develop a set of parametric functions by describing the evolution of the instantaneous intensity and frequency content of ground acceleration considering a filtrated white noise that depends on the magnitude and source-to-site distance $[3,4]$. To carry out this, it is necessary to have a considerable number of accelerograms registered in the site to find statistical parameters as a function of the magnitude and distance and to obtain simulated motions in the site; these types of empirical models are mathematically acceptable but they have a no physical meaning. (5) Finally, the fifth way is based on a physical model that considers the source, wave propagation and site effects; among the models to generate seismic motions of near fault is that proposed by Boore [5]; another alternative model but that is more convenient to generate motions of distant faults is that proposed by Hartzell [6], which uses small registered earthquakes as empirical Green's functions to synthesize large earthquake records. These methods have the advantage of considering a physical model of the source. However, they present uncertainties in the estimate of seismological parameters such as the stress drop, attenuation and others. To apply methods (1), (4) and (5) it is necessary to determine the seismic scenario to be considered in the design; for the method (2) only the intensity to which the motion should be scaled needs to be determined; for the method (3) it is necessary to define the design spectra in the site. The seismic scenario for the generation of the seismic motions of design needs to be defined in terms of the most likely combination of the magnitude, $M$, and the source-to-site distance, $R$, that contribute significantly to the seismic hazard at the site of interest. 
What follows presents the most likely values of magnitude, $M$, and distance, $R$, to generate seismic motions whose intensity value (spectral ordinate) is associated with a given annual frequency of exceedance; these are obtained by means of the application of a method of disaggregation probabilistic seismic hazard assessment,. The method is applied to the case of hard soil at the south of Mexico City. Account is taken of the fact that distinct sources yield very different strong ground motions in the city. Therefore, the following criteria are presented: (i) establishment of structural periods when the spectral ordinates should be used so that the response of the expected motion does not exceed the uniform hazard spectra and (ii) determination of the minimum number of design strong ground motions that must be considered to include spectral ordinates of uniform hazard spectra.

\section{Site and seismic sources}

The growth of Mexico City has originated an important urban development to the south, in which commercial, business, educational and residence centers and bridges are being built. That is why it is important to carry out studies in order to design more economic and reliable structures. South of Mexico City, the site of University City (CU) is considered as the reference site of hard soil, because the CU station has recorded since 1964 more than 20 moderate and intense earthquakes $(M>6.0)$. It has resulted having a better prediction of the expected motion in hard soil of Mexico City.

The seismic risk in Mexico City is related to four sources: subduction, intermediate depth, continental and local earthquakes. All of them have caused economic losses and fatalities. The subduction earthquakes not only take place with more frequency, but they are also particularly those that cause more violent motions in the valley of Mexico, because the type of waves that they produce are rich in long periods that suffer less attenuation and great amplification when crossing the clays of the lake of Mexico City. The earthquakes of intermediate depth are located inside the oceanic plates. Historically the earthquakes of intermediate depth have caused damages in cities and towns in the Mexican antiplane. The magnitude of these earthquakes rarely exceeds a magnitude of 7.0, and their occurrence is much more sporadic. However, due to the location of the source and the proximity to the great populations they may constitute a hazard and high seismic risk to the central population of Mexico. The local and continental earthquakes are the sources that could yield the largest intensities for the hill zone. However, the seismic hazard is small due to the large periods of recurrence for this type of sources ( $>1000$ years). Therefore, subduction and intermediate depth earthquakes are those that represent the maximum perils for the hill zone in Mexico City. Fig. 1 shows the limits and location of the main of subduction and intermediate depth seismic sources idealized by polygons [7]. 


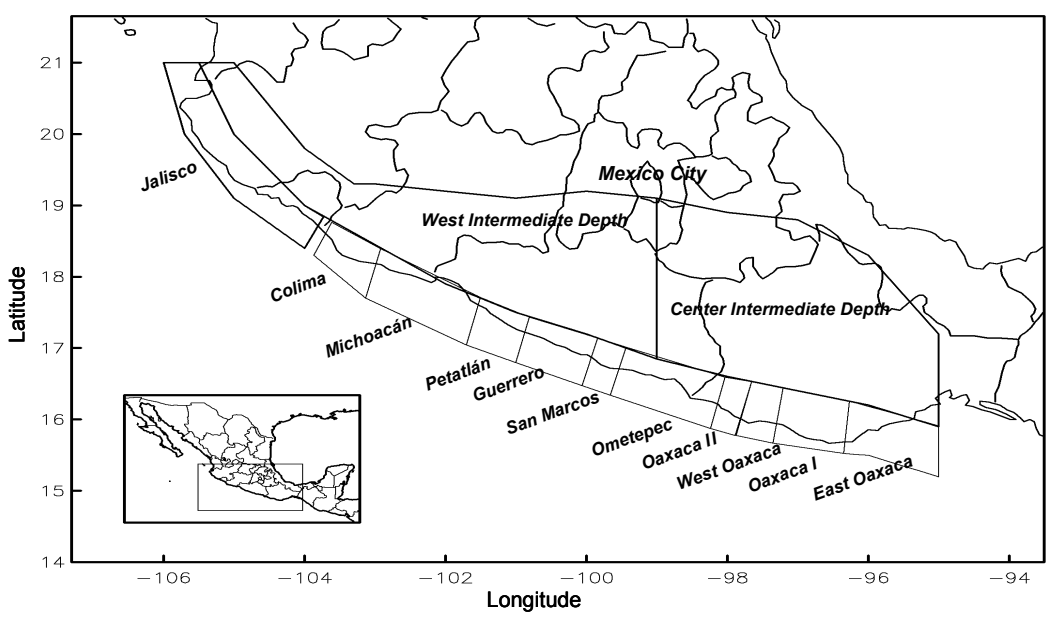

Figure 1: Seismotectonic zones of subduction and intermediate depth used.

\section{Seismic hazards in the site}

For the selection of the seismic design motions it is desirable to rely on a Probabilistic Seismic Hazard Assessment (PSHA) identifying all possible seismic sources that may affect the zone, taking into account all combinations of magnitudes and distances, with the purpose of estimating the exceedance rate, to which some seismic intensity is presented $[8,9]$. This rate is given by

$$
v(y)=\sum_{k} w_{k} \int_{M_{o}}^{M_{u}} f_{M}(m) P(Y>y \mid m, r) \mathrm{d} m
$$

where $w_{k}$ is the sum of the assigned weights of the different seismic sources $k ; M_{o}$ and $M_{u}$ are, respectively, the minimum and maximum magnitude that can be generated in the seismic source $k ; f_{M}(m)$ is the probability density function of the magnitude, $P\left(Y>y \mid m, r_{i, j}\right)$ is the conditional probability function that the seismic intensity, $Y$, exceeds the value of the intensity, $y$, in the site.

For the determination of the seismic hazard, it is necessary to have information on the attenuation of the motions from the source to the site. Three different attenuation laws are used to estimate seismic motions of firm sites in Mexico City. For the subduction earthquakes the relationship used is that derived by Reyes [10] which was built using 22 accelerograms recorded at the station CU. For intermediate depth earthquakes an attenuation law presented by Rosemblueth et al. [11] is used. Finally, for the Tran-Mexican Volcanic Belt, the relation of Singh et al. [12] is used. Fig. 2 shows seismic hazard curves in CU for two structural periods, $T=0.15 \mathrm{~s}$ and $T=2 \mathrm{~s}$ for spectral pseudoacceleration (5\% damping) due to the main seismic sources that affect the site, subduction (thin 
continuous lines), intermediate depth (thick continuous lines) and other seismic sources where events of local or volcanic origin (thin discontinuous lines) are generated and the total contribution to the seismic hazard of all the sources in the site (thick discontinuous lines). The figure shows, that the seismic hazard for structures with period $T=0.15 \mathrm{~s}$ (fig. 2a) is dominated by the sources of intermediate depth (continuous lines) for different exceedance rates, and for structures with period $T=2 \mathrm{~s}$ (fig. $2 \mathrm{~b}$ ) it is dominated by the subduction sources (thin continuous lines).

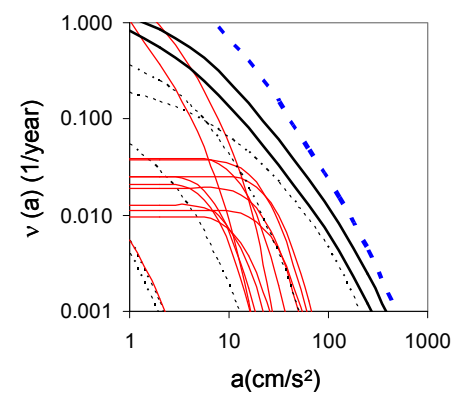

a) Period $0.15 \mathrm{~s}$

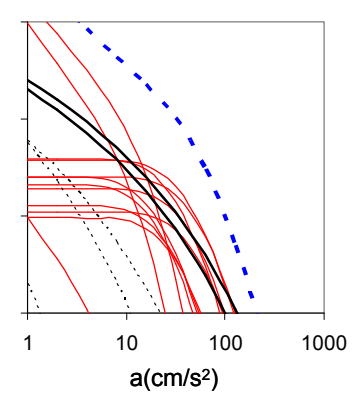

b) Period $2 \mathrm{~s}$

Figure 2: Seismic hazard curves for two structural periods at hard soil in Mexico City.

From the seismic hazard curves it is possible to obtain the uniform hazard spectra associated with an exceedance rate (inverse return period). The uniform hazard spectra represent the seismic ordinates whose intensities have the same probability to occur, considering the contribution of all seismic sources. Fig. 3 shows the uniform hazard spectra (UHS) for a return period of 125 (thin line) and 475 (thick line) years. The UHS shows that for intermediate depth earthquakes the maximum amplitudes are concentrated at periods of less than $1 \mathrm{~s}$, whereas, for the subduction earthquakes the maximum amplitudes are present at periods larger than $1 \mathrm{~s}$. Also, it is observed that there are two maximum ordinates that reflected the seismic hazard dominated by the two types of sources; subduction, in the period $T=1 \mathrm{~s}$ and for intermediate depth, in the period $T=0.15 \mathrm{~s}$.

The result of including different seismic sources impacts in an overestimation of the intensities in the higher modes of vibration when a design is carried out through a spectral modal analysis. This is because the design spectrum in which the uniform hazard spectra are also implicit represents design earthquakes in different seismic sources that occur in a simultaneous way. This means that, the structure is designed to resist different seismic events, but this does not occur, because after the occurrence of the first event at the level of intensity for which the structure was designed, it will be repaired, reinforced or even demolished if required. This means that the buildings should not be designed to resist a series of earthquakes originated at the same time in different seismic sources. Also, the combination of the contribution to the seismic hazard of all the seismic sources for 
a level of intensity that is presented with a frequency of exceedance has the following consequences: (1) the seismic sources that are able to generate the seismic motions associated with the level of intensity, $y$, are ignored; (2) among the seismic sources that can produce such motions, those of them having more possibilities to do so are ignored and (3) due to the existence of a great variety of combinations of magnitudes and distances that can generate this seismic intensity, it is not possible to know what magnitude and what distance of the distinct seismic sources are more likely to generate the motion and this yields the required level of intensity. For these motions selection, the Disaggregation Probabilistic Seismic Hazard Assessment (DPSHA) should be applied with the purpose of identifying the seismic scenarios that contribute more significantly to the seismic hazard and to obtain the seismic motions of design.

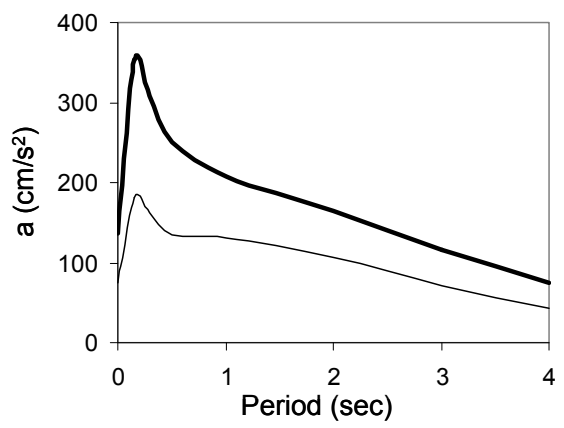

Figure 3: Uniform Hazard Spectra at hard soil in Mexico City for two return periods: 125 (thin line) and 475 (thick line) years.

\section{Disaggregation probabilistic seismic hazard assessment in the site}

Numerous research papers can be useful in obtaining the DPSHA [4, 13-16]. In these papers, the joint conditional probability function of the magnitude and the distance for a level of intensity, $y$, is obtained from a formula given by Chapman [15]:

$$
U(m, r \mid y)_{k}=\sum_{k=1}^{n} \alpha_{k} P(Y \geq y \mid m, r)_{k} f_{M}(m)_{k} f_{R}(r)_{k}
$$

where $U(\bullet)$ is considered as the joint conditional probability density function of $M$ and $R$ for a given value of the intensity, $y ; \alpha_{k}$ is the number of earthquakes with magnitude $m$ between the minimum and maximum values per unit of time; $f_{R}(r)_{k}$ is the probability density function of the distance.

The couple of values of magnitude and distance that more frequently occur (mode) have been calculated and as a result of this, the pairs of values that are more likely to happen. With these values, it is possible to obtain seismic motions 
of design with some of the methods mentioned previously (Methods 1, 4 or 5). Fig. 4 shows the joint conditional probability density function of magnitude and distance for a given value of the intensity associated with a return period of 125 years for the structural periods of $0.15,0.5,1$ and $2 \mathrm{~s}$. In this figure, the modal values of the magnitude, $M$, and distance, $R$, that present the larger probability of occurrence for this return period are also indicated. It can be appreciated that for structural periods $T<1 \mathrm{~s}$ the seismic hazard is dominated by intermediate depth earthquakes (nearest, $R=113 \mathrm{~km}$ ) and for periods $T \geq 1$ s by subduction earthquakes (more distant $R=263 \mathrm{~km}$ ). Therefore, when selecting motions for design whose seismic intensity is associated with a return period in structures with period $T<1 \mathrm{~s}$, they must have characteristics of motion of intermediate depth, mainly a larger content of high frequencies; and for structures with periods $T \geq 1 \mathrm{~s}$ the characteristics of motions of subduction type (larger content of low frequencies). This does not imply that a single motion associated with a level of hazard determines the total behavior that will have the building in its useful life. In order to efficiently analyze and design buildings, engineers should take into account that distinct sources cause very different strong ground motion in the city. For this reason, engineers should consider other types of motions that can occur causing not only damages in the building, but also damages in the contents of the same one due to a larger participation of higher modes of vibration.
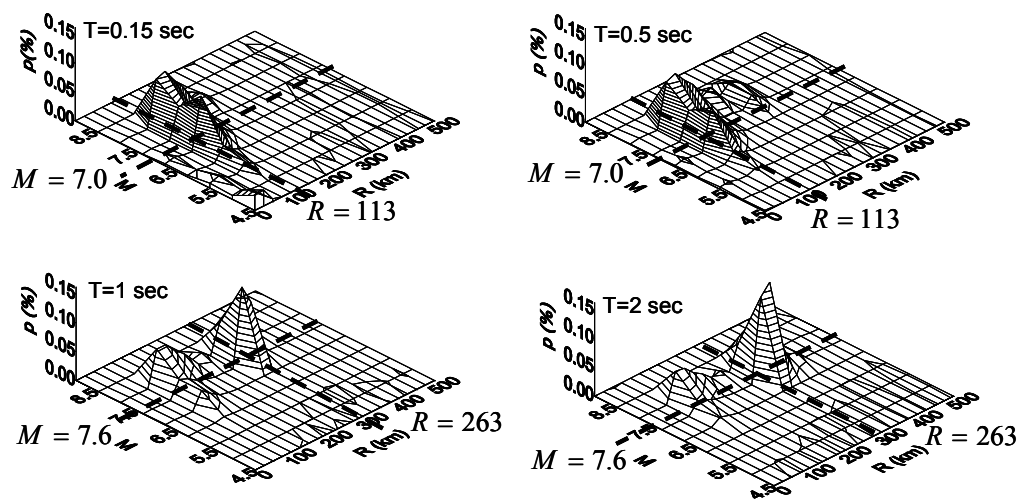

Figure 4: Joint conditional probability density functions of the magnitude and distance when seismic intensity is associated with a return period of 125 years, for different structural periods at hard soil. The pairs of values of magnitude and distance that more probably have to generate this intensity are also indicated.

\section{Criterion of selection of seismic motions for design}

In terms of standard computation of seismic hazard at any site all sources should be taken into account. However, results of the DPSHA show that there is a couple of parameters $M$ and $R$ for all seismic sources that represent the most likely event to generate a required level of intensity associated with an occurrence rate for the 
fundamental period of the structure. In spite of that, it is necessary to consider the occurrence of other events that can occur and affect the behavior of the structure.

Subduction earthquakes have been larger and more frequent than intermediate depth ones, but both represent a threat to Mexico City. For a site such as CU, intermediate depth earthquakes affect structures with period $T<1 \mathrm{~s}$ more significantly than subduction ones. However, some intermediate depth earthquakes may affect structures with period $T>1 \mathrm{~s}$ when the participation of higher modes of vibration has a significant effect on the structural behavior. Therefore, the next three criteria are used to establish for which structural periods the spectral ordinates should be used so that the response of the expected motion does not exceed the uniform hazard spectra and to find the minimum number of design strong ground motions that must be considered for inclusion in the spectral ordinates of uniform hazard spectra.

\subsection{Case 1: structures with period $T<1 \mathrm{~s}$}

For structures with period $T<1 \mathrm{~s}$, the intermediate depth earthquakes could cause heavy damage to small buildings and houses in the hill zone, therefore, at least one of the design motions must be of this type with the magnitude and distance that are more likely (fig. 4) to generate a seismic motion whose spectral ordinate in the period of the structure is associated with a required return period. The second design motion must be also of an intermediate depth, but of such form that the spectral ordinate at the period of $T=0.15 \mathrm{~s}$ is associated with the required return period; because at this period maximum seismic ordinate occurs due to some seismic source of intermediate depth for any return period. And the third seismic motion of some subduction source must be selected, associated with a return rate at the period $T=1 \mathrm{~s}$, because in the indicated period, the maximum seismic ordinate occurs due to some subduction source for any return period (fig. 5a).

\subsection{Case 2: structures with period $T \geq 1 \mathrm{~s}$}

The subduction earthquakes affect more structures with period $T \geq 1 \mathrm{~s}$, because the strong ground motion is long and rich in long periods, therefore, the first design motion must be selected with the characteristics of a subduction source with the magnitude and distance more likely (fig. 4) to generate an event whose spectral ordinate in the period of the structure is associated with the required return period; while the second and third design motions are similar to those of Case 1 (fig. 5b). However, it is important to take into account the second design motion, because intermediate depth earthquakes excite other frequencies that might present a risk for hard soil in Mexico City, especially for small buildings and when the participation of the higher modes of vibration has significant effect on the structural behavior of buildings. Fig. 5 shows the two cases, structure with period $T<1 \mathrm{~s}$ (Case 1, top) and structure with period $T \geq 1 \mathrm{~s}$ (Case 2, bottom). It is observed that for the structure with period $T<1 \mathrm{~s}$ (fig. $5 \mathrm{a}, T=0.3 \mathrm{~s}$ ) the spectral ordinates of interest are matched by three seismic motions of design. And the case is the same for the structure with $T \geq 1 \mathrm{~s}$ (fig. $5 \mathrm{~b}, T=3 \mathrm{~s}$ ). 


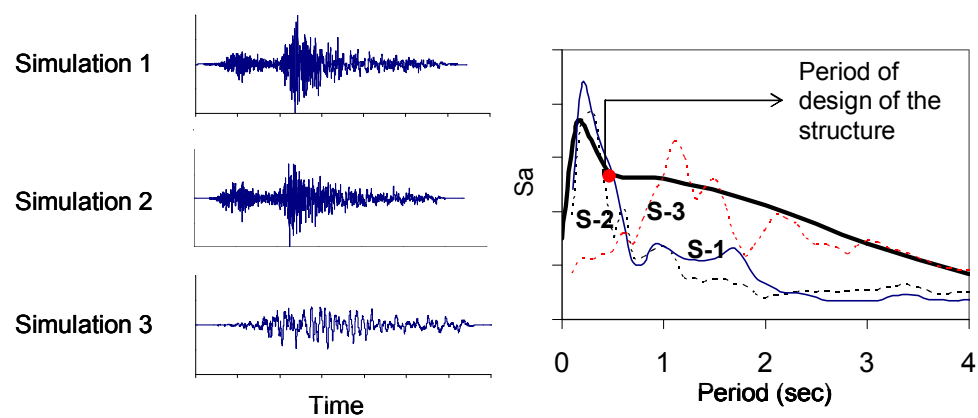

a) Case 1. For structures with period smaller than $1 \mathrm{~s}$
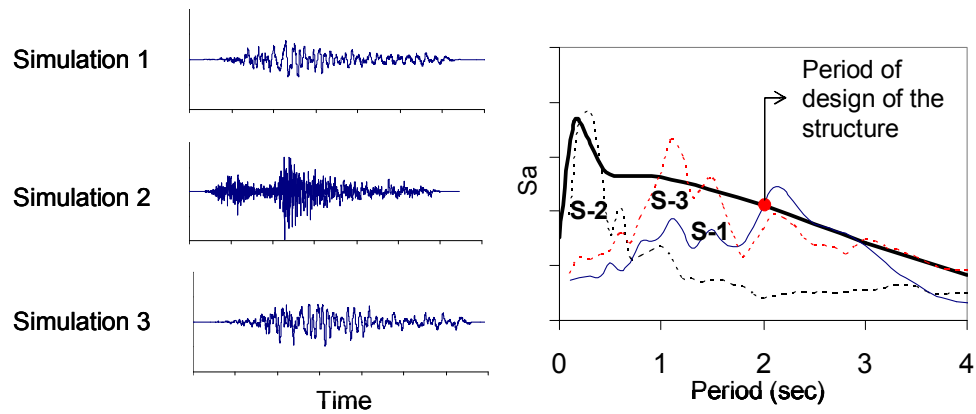

b) Case 2. For structures with period larger than $1 \mathrm{~s}$

Figure 5: Motions of design and response spectra for the proposed Cases 1 and 2.

\section{Conclusions}

By means of the application of a method of disaggregation probabilistic seismic hazard in hard soil, it is possible to find the magnitude and distance which are more likely to generate an intensity associated with a period of return of 125 years in Mexico City. The main seismic sources that affect more structures on hard soil are determined. It is also determined what structural periods should be associated with the spectral ordinates so that the response of the expected motions in hard soil does not exceed those obtained with the uniform hazard spectra. It is found that for structures with period $T<1 \mathrm{~s}$ the design motions should be generated from one source of intermediate depth, and for structures with $T \geq 1 \mathrm{~s}$ the motions should be generated from one subduction source. It is also shown that three minimum motions must be considered in the design to match relatively well the intensities of the uniform hazard spectra. These motions must be generated for a spectral ordinate associated with a return rate for the following periods: (1) for $T=0.15 \mathrm{~s}$, the motion must be produced from an intermediate depth source, (2) for $T=1 \mathrm{~s}$, it must be produced from a subduction source and (3) for a period $T$ similar to the period of the structure of interest. 


\section{References}

[1] Bommer, J.J., Scott, S.G. \& Sarma, S.K., Hazard-consistent earthquake scenarios. Soil Dynamics and Earthquake Engineering, 19, pp. 219-231, 2000.

[2] Naeim, F. \& Lew, M., On the use of the design spectrum compatible time histories. Earthquake Spectra, 11(1), pp. 111-127, 1995.

[3] Nojima, N. \& Kameda, H., Simulation of risk-consistent earthquake motion. Proc. of Ninth World Conference on Earthquake Engineering, II, pp. 95-100, 1988.

[4] Alamilla, J., Esteva, L., García, P.J. \& Díaz, L.O., Simulating earthquake ground motion at a site, for given intensity and uncertain source location. Journal of Seismology, 5(4), pp. 475-485, 2001.

[5] Boore, D.G., Stochastic simulation of high-frequency ground motions based on seismological models of the radiated spectra. Bulletin of the Seismological Society of America, 73, pp. 1865-1894, 1983.

[6] Hartzell, S., Earthquake aftershocks as Green's functions. Geophysical Research Letters, 5, pp. 1-4, 1978.

[7] Nishenko, S.P. \& Singh, S.K., The Acapulco-Ometepec, México earthquake of 1907-1982: Evidence for a variable recurrence history. Bulletin of the Seismological Society of America, 77, pp. 1359-1367, 1987.

[8] Cornell, C., Engineering seismic risk analysis. Bulletin of the Seismological Society of America, 58(5), pp. 1583-1606, 1968.

[9] Esteva, L., Regionalización sísmica de México para fines de ingeniería. Series del Instituto de Ingeniería, 246, 1970.

[10] Reyes, C., El estado limite de servicio en el diseño sísmico de edificios. Ph. D. Thesis, Faculty of Engineering, UNAM, 1999.

[11] Rosemblueth, E., Ordaz, M., Sanchez-Sesma, F. \& Singh, S.K., Design spectra for Mexico's Federal District. Earthquake Spectra, 5, pp. 273-291, 1989.

[12] Singh, S.K., Santoyo, M. \& Pacheco, J.F., Intermediate-depth earthquake in central Mexico: Implications for plate waves. Geophysical Research Letters, 22, pp. 527-530, 1995.

[13] Ishikawa, Y. \& Kameda, H., Hazard-consistent magnitude and distance for extended seismic risk analysis. Proc. of Ninth World Conference on Earthquake Engineering, II, pp. 89-94, 1988.

[14] McGuire, R., Probabilistic seismic hazard analysis and design earthquakes: closing the loop. Bulletin of the Seismological Society of America, 85, pp. 1275-1284, 1995.

[15] Chapman, M., A probabilistic approach to ground-motion selection for engineering design. Bulletin of the Seismological Society of America, 85(3), pp. 937-942, 1995.

[16] Bazurro, P. \& Cornell, C., Disaggregation of seismic hazard. Bulletin of the Seismological Society of America, 89(2), pp. 501-520, 1999. 